\title{
How many species of whipworms do we share? Whipworms from man and other primates form two phylogenetic lineages
}

\author{
Jana Doležalová ${ }^{1,2,3, \pm}$, Miroslav Oborník ${ }^{4,5, \pm}$, Eva Hajdušková ${ }^{4}$, Milan Jirkü ${ }^{4}$, Klára J. Petrželková2,4,6,7, Petra \\ Bolechová $^{7,8}$, Cristina Cutillas $^{9}$, Rocio Callejón ${ }^{9}$, Jozef Jaroš ${ }^{10}$, Zuzana Beránková ${ }^{11}$ and David Modrý $\dot{y}^{2,3,4}$
}

\author{
${ }^{1}$ Department of Physiology, Faculty of Veterinary Medicine, University of Veterinary and Pharmaceutical Sciences, Brno, Czech \\ Republic; \\ ${ }^{2}$ Department of Pathology and Parasitology, Faculty of Veterinary Medicine, University of Veterinary and Pharmaceutical Sciences, \\ Brno, Czech Republic; \\ ${ }^{3}$ CEITEC - Central European Institute of Technology, University of Veterinary and Pharmaceutical Sciences, Brno, Czech Republic; \\ ${ }^{4}$ Institute of Parasitology, Biology Centre of the Czech Academy of Sciences, České Budějovice, Czech Republic; \\ ${ }^{5}$ Faculty of Science, University of South Bohemia, České Budějovice, Czech Republic; \\ ${ }^{6}$ Institute of Vertebrate Biology of the Czech Academy of Sciences, Brno, Czech Republic; \\ ${ }^{7}$ Liberec Zoo, Liberec, Czech Republic; \\ ${ }^{8}$ Department of Husbandry and Ethology of Animals, Czech University of Life Sciences Prague, Czech Republic; \\ ${ }^{9}$ Department of Microbiology and Parasitology, Faculty of Pharmacy, Sevilla University, Sevilla, Spain; \\ ${ }^{10}$ Department of Histology and Embryology, Faculty of Medicine, Masaryk University, Brno, Czech Republic; \\ ${ }^{11}$ BioTest s.r.o., Konárovice, Czech Republic \\ ${ }^{ \pm}$These author contributed on the work equally.
}

\begin{abstract}
The whipworms, i.e. parasitic nematodes of the genus Trichuris Roederer, 1761, infect a variety of mammals. Apparently low diversity of primate-infecting species of Trichuris strongly contrasts with the high number of species described in other mammalian hosts. The present study addresses the diversity of whipworms in captive and free-ranging primates and humans by analysing nuclear (18S rRNA, ITS2) and mitochondrial (coxl) DNA. Phylogenetic analyses revealed that primate whipworms form two independent lineages: (i) the Trichuris trichiura (Linnaeus, 1771) clade comprised of genetically almost identical whipworms from human and other primates, which suggests the ability of $T$. trichiura to infect a broader range of primates; (ii) a clade containing primarily Trichuris suis Schrank, 1788, where isolates from human and various primates formed a sister group to isolates from pigs; the former isolates thus may represent of more species of Trichuris in primates including humans. The analysis of coxl has shown the polyphyly of the genera Trichuris and Capillaria, Zeder, 1800. High sequence similarity of the T. trichiura isolates from humans and other primates suggests their zoonotic potential, although the extent of transmission between human and other non-human primates remains questionable and requires further study.
\end{abstract}

Keywords: Trichuris, phylogeny, diversity, zoonotic potential, humans

This article contains supporting information (Figs. S1-S5) online at http://folia.paru.cas.cz/suppl/2015-62-063.pdf

The nematode genus Trichuris Roederer, 1761 includes more than 60 species that parasitise various mammalian species from the orders Artiodactyla, Carnivora, Didelphimorphia, Lagomorpha, Peramelemorphia, Primates and Rodentia (Anderson 2000). For decades, taxonomic studies almost exclusively focused on whipworm species from ruminants or rodents (e.g. Knight 1983, Robles 2011, Torres et al. 2011), probably due to better accessibility to material from these hosts.

Whipworm infections are common in many species of free-ranging primates in Africa (e.g. Ashford et al. 2000, Kalema-Zikusoka et al. 2005), America (Michaud et al. 
2003, Phillips et al. 2004) and Asia (Mul et al. 2007, Labes et al. 2010) as well as those in captivity (Melfi and Poyser 2007, Lim et al. 2008). Trichuris lemuris Rudolphi, 1819 and Trichuris cynocephalus Khera, 1951 are together with Trichuris trichiura (Linnaeus, 1771) species described from primates (Khera 1951, Chabaud et al. 1964). Since infections with whipworms in primates were usually diagnosed as those caused by T. trichiura (see Ooi et al. 1993, Reichard et al. 2008, Lee et al. 2010), several authors have expected zoonotic cross-transmission between non-human primates and humans (Munene et al. 1998, Chapman et al. 2006).

Such an unusually broad host range of $T$. trichiura raises the questions of host specificity and possibility of underestimated diversity of species in non-human primates. The low diversity of species of Trichuris in primates strongly contrasts with the high number of those found in other mammalian hosts. The assemblage of species of Trichuris infecting rodent hosts is probably the most diversified one, with 21 species reported from rodents of nine families in North and South America (Robles 2011). Another 11 species of Trichuris parasitise four families of ruminants (e.g. Knight 1983, Cutillas et al. 2004).

The exact species determination of Trichuris spp. based solely on egg morphology is virtually impossible (Špakulová 1994) and adult worms are difficult to obtain from primates. Thus, molecular taxonomy offers a powerful approach for the evaluation of diversity of trichurid species in these hosts. During the past decades, nuclear (ITS1-5.8SITS2) and mitochondrial (coxl) DNA have been used for species identification, as well as for phylogenetic analyses of species of Trichuris from rodents, pigs and cattle (Cutillas et al. 2004, 2009, Callejón et al. 2009, 2012).

Recently, the analyses of sequences of the coxl gene of mitochondrial DNA have confirmed that Trichuris suis Schrank, 1788 and T. trichiura represent two distinct species (Liu et al. 2012). Interestingly, studies using ITS2 sequence have also claimed that isolates of T. trichiura from humans and non-human primates form two distinct clades, although the interpretations of two studies were different. Whereas Nissen et al. (2012) found in humans two types of clones of T. trichiura (one similar to T. trichiura and another similar to T. suis) and heterozygotes that have both types of rDNA, Ravasi et al. (2012) concluded that these findings were consequence of cross-contamination of DNA and showed on their own isolates the suggested existence of distinct species of Trichuris sp. in human and non-human primates.

The present study contributes to a better understanding of the diversity of species of Trichuris in humans and non-human primates using phylogenetic analyses combining both nuclear and mitochondrial genes. Moreover, the hypothesised existence of more species of Trichuris in primates opens the possibility to revise the zoonotic potential and host specificity of T. trichiura and other, putative new species of whipworms.

\section{MATERIALS AND METHODS}

\section{Study sites and sampled hosts}

Faecal samples containing the eggs of whipworms (detected by coproscopic techniques, see below) were obtained from several captive primate species including chimpanzee, Pan troglodytes Blumenbach, yellow-cheeked gibbon, Nomascus gabriellae Thomas, olive baboon, Papio anubis Lesson, and gelada baboon, Theropithecus gelada Rüppell, lion-tailed macaque, Macaca silenus Linnaeus, green monkey, Chlorocebus sabaeus Linnaeus and rhesus macaque, Macaca mulatta Zimmermann. Adult whipworms were acquired during necropsy from hamadryas baboon, Papio hamadryas Linnaeus, long-tailed macaque, Macaca fascicularis Raffles, and eastern black-and-white colobus monkey, Colobus guereza kikuyuensis Lönnberg; nematodes from the latter host were in the meantime described as Trichuris colobae Cutillas, Oliveros et Callejón, 2014.

Faecal samples from free-ranging primates were acquired from two sites: one isolate from a wild chimpanzee residing in the Cantanhez National Park, Guinea-Bissau; six samples from a chimpanzee, vervet monkeys, Chlorocebus aethiops pygerythrus Cuvier, and black-and-white colobus monkey, Colobus guereza guereza Rüppell, living in the Rubondo Island National Park, Tanzania. Finally, a single adult female of Trichuris was obtained from an anonymous Czech researcher.

In addition, eggs of whipworms isolated from domestic pig, Sus scrofa Linnaeus, domestic dog, Canis lupus Linnaeus, and Bactrian camel, Camelus bactrianus Linnaeus, were included into the study (summary of all isolates in Table 1).

\section{Sample collection and preparation}

Faecal samples from captive animals were collected immediately after defecation and preserved in $20 \mathrm{ml}$ of $10 \%$ formalin $(2-5 \mathrm{~g})$ and $5 \mathrm{ml}$ of $96 \%$ ethanol $(\sim 2 \mathrm{~g})$, the samples from free-ranging chimpanzees, colobus and vervet monkeys were collected as described in Petrášová et al. (2010) and Sá et al. (2013). The adult whipworms were acquired by colonoscopy (Homo sapiens, isolate 1) or necropsy of hosts (Macaca fascicularis - isolate 9, Papio hamadryas - isolate 7, Colobus guereza kikuyuensis - isolate 12) and also preserved in $96 \%$ ethanol.

The flotation technique with modified Sheather's solution (Sheather 1923) was used for initial coproscopic analysis of formalin-preserved samples. In cases where whipworm eggs were present (Fig. 1), ethanol preserved samples were processed by flotation technique as well. The obtained eggs were collected from the surface of the flotation solution using a horizontal wire loop and transferred into a $10 \mathrm{ml}$ vial with $8 \mathrm{ml}$ of phosphate buffered saline (PBS). Thereafter, the vial was centrifuged $2 \mathrm{~min}$ at $\sim 320 \times g$ and the pellet containing eggs was suspended in $1 \mathrm{ml}$ of PBS; $40 \mu 1$ was pipetted onto a microscopic slide using a dimple.

Individual eggs were sucked up by a thin glass micropipette (normally used for embryo transfers) equipped with a short silicone hose having an inner diameter of $2 \mathrm{~mm}$ and a node on one end. The eggs (minimum 30) were transferred into $0.5 \mathrm{ml}$ of PBS in a $2 \mathrm{ml}$ micro tube. Prior to DNA extraction, the egg shells were disrupted with glass beads $(0.5$ and $1 \mathrm{~mm})$ using a BeadBeater 
Table 1. The locality, source of DNA and GenBank accession numbers of particular genes of whipworm isolates acquired from non-human primates and humans. The extended data set includes isolates from a domestic pig, dog and Bactrian camel. The whipworm isolates from colobus monkeys $(13,14$ and 15$)$ and chimpanzees $(3,4)$ were identical in the $18 \mathrm{~S}$ rRNA gene sequences with that from gelada baboon.

\begin{tabular}{|c|c|c|c|c|c|c|}
\hline Isolate & Host & Locality & Source of DNA & $\operatorname{cox} 1$ & 18S'rDNA & ITS2 \\
\hline 1 & Homo sapiens & Brno, CZ & adult & JF690962 & JF690953 & JF690940 \\
\hline 2 & Pan troglodytes Blumenbach & Antwerp Zoo, NL & eggs & - & - & JF690948 \\
\hline 3 & Pan troglodytes & Rubondo NP, EAT & eggs & - & + & - \\
\hline 4 & Pan troglodytes & Cantanhez NP, GW & eggs & - & + & - \\
\hline 5 & Nomascus gabriellae Thomas & Bratislava Zoo, SK & eggs & - & - & JF690947 \\
\hline 6 & Papio anubis Lesson & Brno Zoo, CZ & eggs & JF690964 & JF690955 & JF690942 \\
\hline 7 & Papio hamadryas Linnaeus & Liberec Zoo, CZ & adult & JF690963 & JF690954 & JF690941 \\
\hline 8 & Theropithecus gelada Rüppell & Brno Zoo, CZ & eggs & JF690965 & JF690956 & JF690943 \\
\hline 9 & Macaca fascicularis Raffles & Konárovice, $\mathrm{CZ}$ & adult & JF690967 & JF690958 & JF690946 \\
\hline 10 & Macaca mulatta Zimmermann & Konárovice, $\mathrm{CZ}$ & eggs & - & JX049339 & - \\
\hline 11 & Macaca silenus Linnaeus & Liberec Zoo, CZ & eggs & JF690966 & JF690957 & JF690945 \\
\hline 12 & Colobus guereza kikuyuensis Lönnberg & Fuengirola Zoo, E & adult & JF690968 & JF690959 & FM991956* \\
\hline 13 & Colobus guereza Rüppell & Rubondo NP, EAT & eggs & - & + & - \\
\hline 14 & Colobus guereza & Rubondo NP, EAT & eggs & - & + & - \\
\hline 15 & Colobus guereza & Rubondo NP, EAT & eggs & - & + & - \\
\hline 16 & Chlorocebus sabaeus Linnaeus & Liberec Zoo, CZ & eggs & - & JX049338 & JF690944 \\
\hline 17 & Chlorocebus aethiops pygerythrus Cuvier & Rubondo NP, EAT & eggs & - & - & JF690949 \\
\hline 18 & Chlorocebus aethiops pygerythrus & Rubondo NP, EAT & eggs & - & - & JF690950 \\
\hline 19 & Sus scrofa Linnaeus & Okoč, SK & eggs & JF690969 & JF690960 & JF690951 \\
\hline 20 & Canis lupus Linnaeus & Brno, CZ & eggs & - & JX049340 & - \\
\hline 21 & Camelus bactrianus Linnaeus & Brno Zoo, CZ & eggs & JF690970 & JF690961 & JF690952 \\
\hline
\end{tabular}

Amplified genes of particular isolates have sign '+' or GenBank Accession Number, not amplified genes are signed by '-'. CZ - Czech Republic, E - pain, EAT - Tanzania, GW - Guinea-Bissau, NL - Netherlands, NP - National Park, SK - Slovak Republic. * Cutillas et al. (2009).

(Biospec Products Inc., Bartlesville, Oklahoma, USA) for $10 \mathrm{~min}$ at a shaking frequency of 2100 oscillations $/ \mathrm{min}$.

The adult whipworms were washed $5 \times$ in PBS, disintegrated by micro-pestles and washed $5 \times$ in PBS, followed by centrifugation for $5 \mathrm{~min}$ at $\sim 16000 \times \mathrm{g}$. After the last centrifugation, the supernatant was removed and the pellet was prepared for DNA extraction.

\section{DNA extraction}

Before DNA extraction, $800 \mu$ l of NET buffer $\mathrm{pH} 8.0$ (4 M $\mathrm{NaCl}, 0.5 \mathrm{M}$ EDTA and $1 \mathrm{M}$ TRIS), $240 \mu \mathrm{l}$ of $30 \% \mathrm{~N}$-lauroylsarcosine sodium salt solution (Sigma-Aldrich, Praha, Czech Republic) and $30 \mu \mathrm{l}$ of proteinase K (Chemos CZ, Praha, Czech Republic) were added to the prepared samples. The homogenate was incubated in a dry bath at $56^{\circ} \mathrm{C}$ for $15 \mathrm{~h}$ and subsequently extracted using the phenol-chloroform method based on the protocol outlined by Sambrook and Russell (2006). The phenol-chloroform extraction yielded DNA at concentrations of $10-15 \mathrm{ng} / \mu \mathrm{l}$ from eggs and $30-130 \mathrm{ng} / \mu \mathrm{l}$ from adults.

\section{PCR amplification and sequencing}

The partial ITS2 and 18S of ribosomal RNA (rDNA) and coxl of mitochondrial DNA (mtDNA) were amplified by PCR using a Biometra thermocycler (Biometra, Göttingen, Germany). For ITS2 (600 bp), the forward primer Proteo1 (5'-CGGTGGATCACTCGGCTC-3'; Škeříková et al. 2004) and reverse primer ITS4 (5'-TCCTCCGCTTATTGATATGC-3'; White et al. 1990) were used with the following PCR conditions: an initial denaturation at $96^{\circ} \mathrm{C}$ for $5 \mathrm{~min}$ and then 30 cycles of $1 \mathrm{~min}$ at $94{ }^{\circ} \mathrm{C}, 1 \mathrm{~min}$ at $56^{\circ} \mathrm{C}$ and $1 \mathrm{~min}$ at $72^{\circ} \mathrm{C}$, followed by $10 \mathrm{~min}$ at $72^{\circ} \mathrm{C}$. Genus-specific primers were designed to amplify a $550 \mathrm{bp}$ fragment of 18S rDNA: forward primer TF (5'-GATGTCCACTTGGATAACTA-3') and reverse primer TR (5'-GACGGAACGACTCCTGCTTA-3'). PCR was performed using the following conditions: an initial denaturation at $96^{\circ} \mathrm{C}$ for $3 \mathrm{~min}$ and then 30 cycles of $1 \mathrm{~min}$ at $94^{\circ} \mathrm{C}, 1 \mathrm{~min}$ at $58^{\circ} \mathrm{C}$ and $1 \mathrm{~min}$ at $72{ }^{\circ} \mathrm{C}$, followed by $10 \mathrm{~min}$ at $72^{\circ} \mathrm{C}$. Finally, a $419 \mathrm{bp}$ fragment of the coxl gene was amplified using the forward primer L6625 (5'-TTYTGRTTYTTYGGNCAYCC-3') and reverse primer H7005 (5'-ACTACGTAGTAGGTATCATG-3'), which were together with PCR conditions modified by Callejón et al. (2009). Each PCR mixture included: $12.5 \mu 1$ of commercial Combi PPP Master Mix (Top-Bio, Praha, Czech Republic), $9.5 \mu 1$ of PCR water, $1 \mu \mathrm{l}$ of each primer mix $(0.01 \mathrm{mM}$ each $)$ and $1 \mu \mathrm{l}$ of template DNA. The PCR products were checked on an ethidium bromide-stained $1 \%$ agarose gel. PCR products were purified from the agarose gel using the QuickClean 5M Gel Extraction Kit (GenScript USA, Inc., Piscataway Township, New Jersey, USA) according to manufacturer's protocol and sequenced at Macrogen Inc. (Seoul, Korea). The nucleotide sequences obtained in our study have been deposited in GenBank under accession numbers FJ690940-FJ690970 and JX049338-JX049340.

To verify the possible presence of paralogs in the genome of species of Trichuris, primers according to Logan et al. (1992) were used to amplify the complete sequence of the $5.8 \mathrm{~S}$ ribosomal RNA gene (rDNA) surrounded by partial sequences of internal transcribed spacers 1 and 2. For this purpose, we have randomly chosen the isolate of Trichuris sp. from Papio hamadryas residing in the Liberec Zoo. Obtained PCR products were cloned and six were sequenced in both directions. No paralogs were detected (data not shown). 

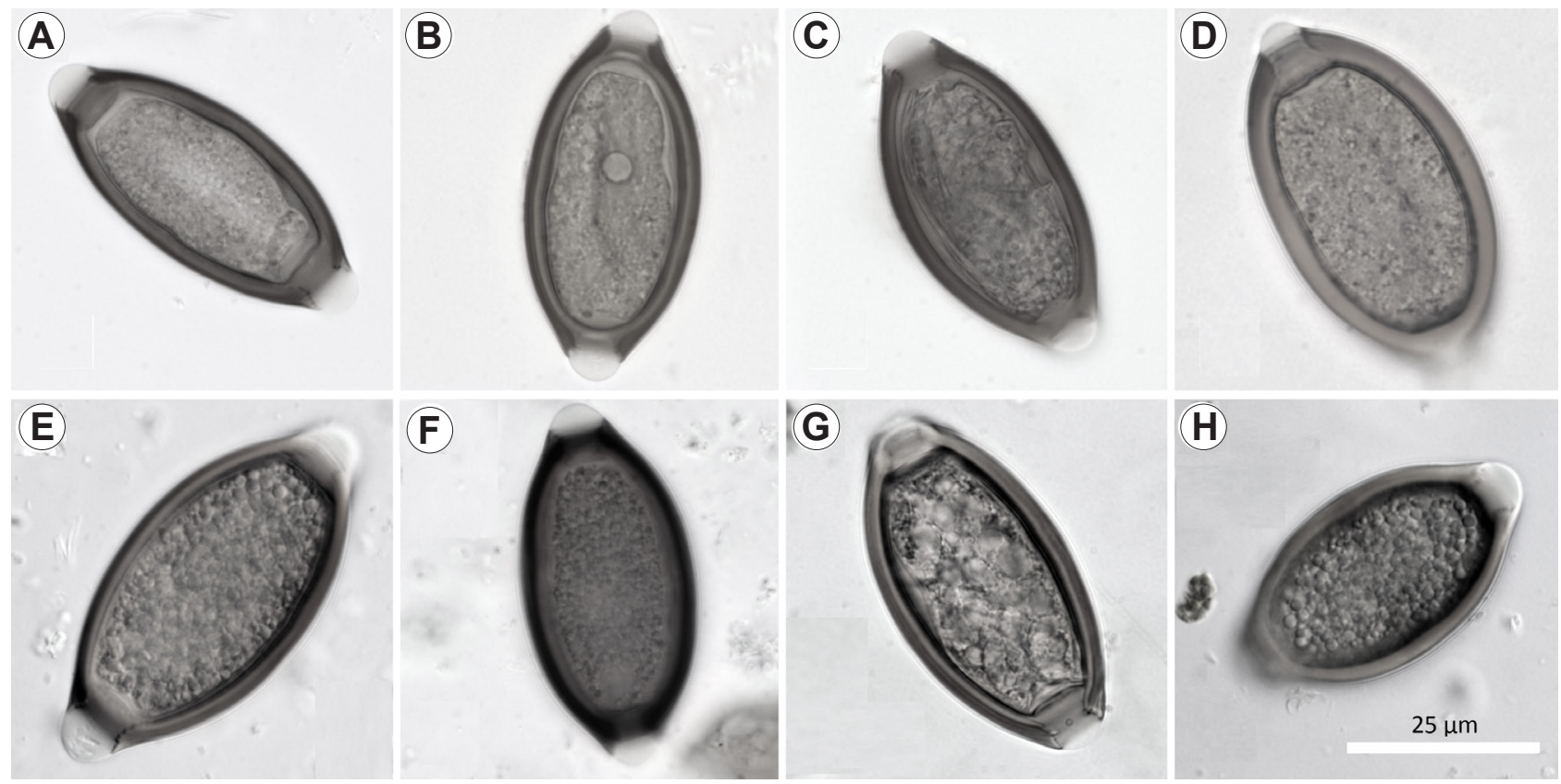

Fig. 1A-H. The variability of the eggs of Trichuris spp. retrieved from the faeces of primates and a domestic pig; Nomarski interference contrast with an Olympus AX70 microscope; all at the same scale. A - Trichuris trichiura (Linnaeus, 1771) from human patient (isolate 1); B - Trichuris sp. from Macaca fascicularis (isolate 9); C - Trichuris sp. from Chlorocebus aethiops pygerythrus (isolate 18); D - Trichuris sp. from Chlorocebus aethiops pygerythrus (isolate 17); $\mathbf{E}$ - Trichuris suis from domestic pig (isolate 19); $\mathbf{F}-$ Trichuris sp. from Macaca silenus (isolate 11); G - Trichuris sp. from Pan troglodytes (isolate 4); $\mathbf{H}$ - Trichuris sp. from Chlorocebus sabaeus (isolate 16).

\section{Phylogenetic analyses}

We used sequences from three different genomic regions to infer phylogenetic relationships among species of Trichuris and related taxa, namely nuclear ITS2 region of the rRNA gene, part of the small subunit rRNA gene (18S rDNA) and the mitochondrial gene coding for coxl. Nuclear genes were aligned using their nucleotide sequence whereas inferred amino acid sequence data were used for cox 1 .

Thus, we aligned 77 ITS2 GenBank ${ }^{\mathrm{TM}}$ sequences of Trichuris spp. from human and animal hosts with an additional 13 newly obtained sequences from humans, non-human primates, pig and camel (Table 1) using the program Kalign (Lassmann and Sonnhammer 2005). The same program was used to align $3818 \mathrm{~S}$ rDNA genes of species of the genus Trichuris, of which 17 were newly obtained. Gaps and ambiguously aligned positions were removed using BioEdit (Hall 1999). Due to high number of gaps, the alignments of ITS2 and partial 18S rDNA gene sequences were also constructed using webPRANK (http://www.ebi.ac.uk/ goldman-srv/webprank/) probabilistic approach and the alignments were edited by Gblocks (Castresana 2000, Talavera and Castresana 2007) in the frame of SEAVIEW program (Gouy et al. 2010).

Phylogenetic trees were computed by maximum likelihood (ML), maximum parsimony (MP) and Bayesian inference (BI) using PhyML (Guindon and Gascuel 2003), PAUP 4b10 (Swofford 2002) and PhyloBayes 3.2 (Ronquist et al. 2012), respectively. ML trees were calculated under the gamma corrected GTR evolutionary model $(\mathrm{GTR}+\mathrm{G})$. Bootstrap support of ML and MP trees was inferred from 1000 replicates. Bayesian topologies and posterior probabilities (PP) were assessed under the gammacorrected GTR substitutional matrix $(\mathrm{GTR}+\mathrm{G})$ using MrBayes 3.2
(Ronquist et al. 2012). Two independent Monte-Carlo Markov chains were allowed to run (under the default settings) for three million generations, of which the first $5 \times 10^{5}$ were omitted from PP (and topology) reconstruction. The ITS2 tree was rooted to Trichuris skrjabini Baskakov, 1924 (AJ489248 - outgroup); the 18S tree was rooted to Trichuris muris (Schrank, 1788) (AF036637 - outgroup). The ML trees were computed using alignments constructed by webPRANK only (see Figs. S1, S2).

We also constructed two trees (for intraspecific and interspecific variability) inferred from coxl amino acid datasets by Neighbor Joining (NJ), MP and ML methods using AsaturA (Van de Peer et al. 2002; AsaturA is a program designed to deal with amino acid saturation), PAUP 4b10 (Swofford 2002) and PhyML (Guindon and Gascuel 2003) programs, respectively. AsaturA was used to avoid phylogenetic artifacts, because sequences HQ204213, HQ204211, HQ204212, AJ288166, AJ288168 and $\mathrm{AJ} 288169$, and new sequences from isolates 9 and 11 constituted long branches in the tree.

For testing intraspecific variability the dataset comprised 44 coxl sequences that are proposed to belong to the genus Trichuris (including 9 new sequences). For testing interspecific variability, this dataset was enriched by sequences of species of the related genera Capillaria Zeder, 1800 (10 sequences) and Anatrichosoma Swift, Boots et Miller, 1922 (1 sequence). Sequences were aligned by Kalign (Lassmann and Sonnhammer 2005), and the alignment was manually verified and edited in BioEdit (Hall 1999); NJ and ML trees were computed using the LG model that was gamma corrected in the case of $\mathrm{ML}(\mathrm{LG}+\mathrm{G}+\mathrm{I})$. Bootstrap supports were computed from 1000 replicates.

Furthermore, a combined dataset of the three gene regions (for which data were available) was used to estimate the deep phy- 


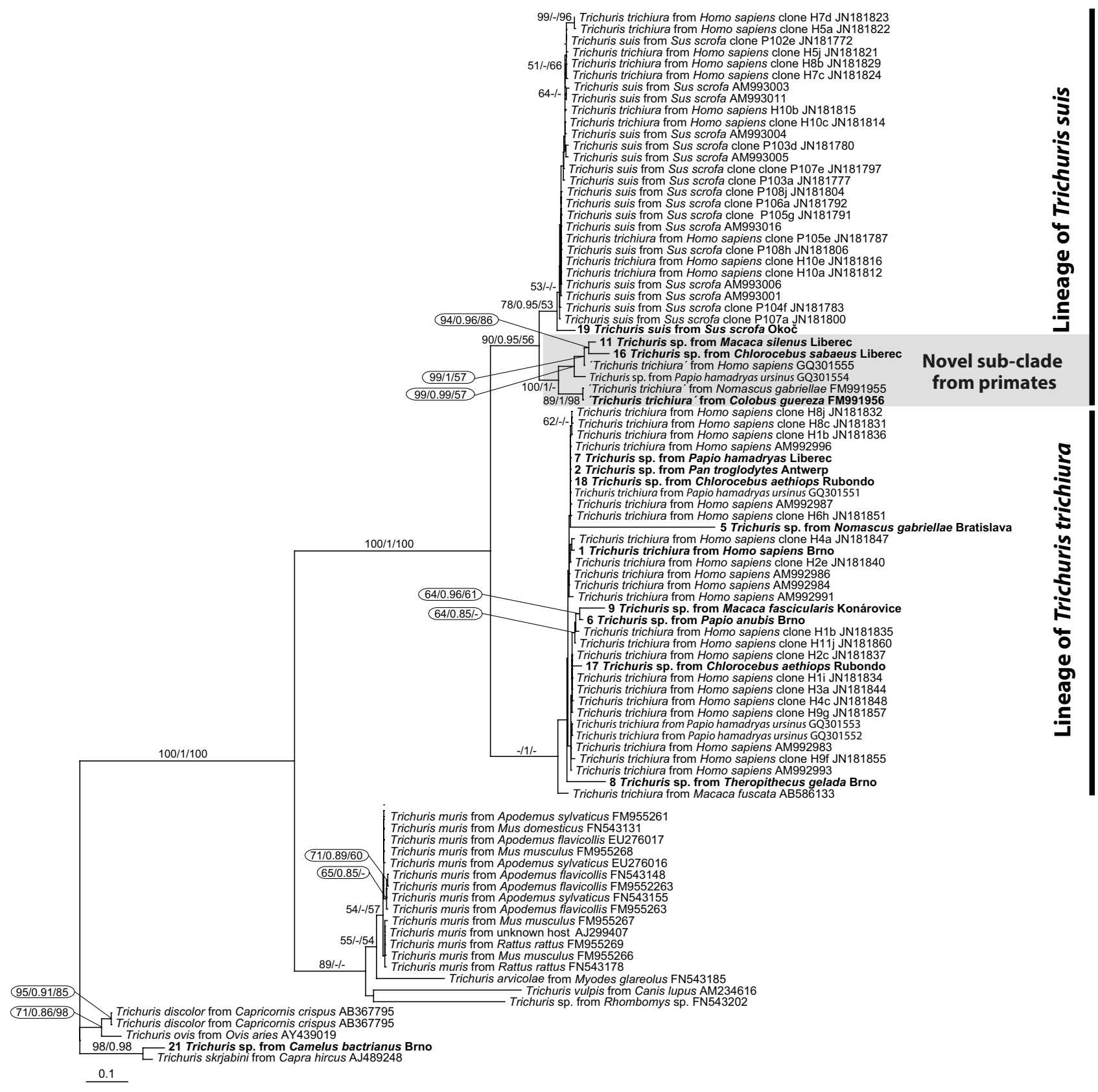

Fig. 2. Maximum likelihood phylogenetic tree as inferred from ITS2 sequences. The tree was computed using the GTR model with discrete gamma distribution in four categories. Numbers above branches indicate ML bootstrap support (1000 replicates)/Bayesian posterior probabilities (PhyloBayes; see Methods for details)/MP bootstrap support (1000 replicates). Sequences newly reported in this study are bold typed. The three basic clades of genus Trichuris are highlighted. Tree was rooted to Trichuris skrjabini Baskakov, 1924 (AJ489248).

logenetic positions of Trichuris isolates. For this purpose we used Trichuris isolates from Sus scrofa (isolate 19), Camelus bactrianus (isolate 21), Colobus guereza (isolate 12), Macaca silenus (isolate 11), M. fascicularis (isolate 9), Papio anubis (isolate 6), P. hamadryas (isolate 7), Theropithecus gelada (isolate 8) and Homo sapiens (isolate 1). These were studied together with Trichuris trichiura (2 samples), T. suis (4 samples), T. arvicolae Feliu, Morand et Hugot, 2000 (1 sample), T. muris (1 sample), T. ovis (Abildgaard, 1795) (1 sample), T. discolor (von Linstow, 1906) (1 sample) and T. skrjabini (1 sample) sequences deposited in the GenBank database. The nucleotide alignment of individual gene was constructed separately using Kalign (Lassmann and
Sonnhammer 2005); inferred coxl amino acid sequences were aligned in BioEdit.

All three gene regions were consequently concatenated into a single dataset. ML and $\mathrm{BI}$ trees were computed using RAxML 8.1a (Stamatakis 2014) and MrBayes 3.2 (Ronquist et al. 2012), respectively. The dataset was analysed as partitioned (i.e. the model parameters were estimated separately for each respective gene) under the gamma-corrected GTR (GTR + G) (ML and BI) and HKY models (BI). Bootstrap supports of ML tree were computed from 1000 replicates. In BI, two independent chains were run for $3 \times 10^{6}$ generations and posterior probabilities (as a mean of branching support) were estimated after first $1 \times 10^{5}$ genera- 


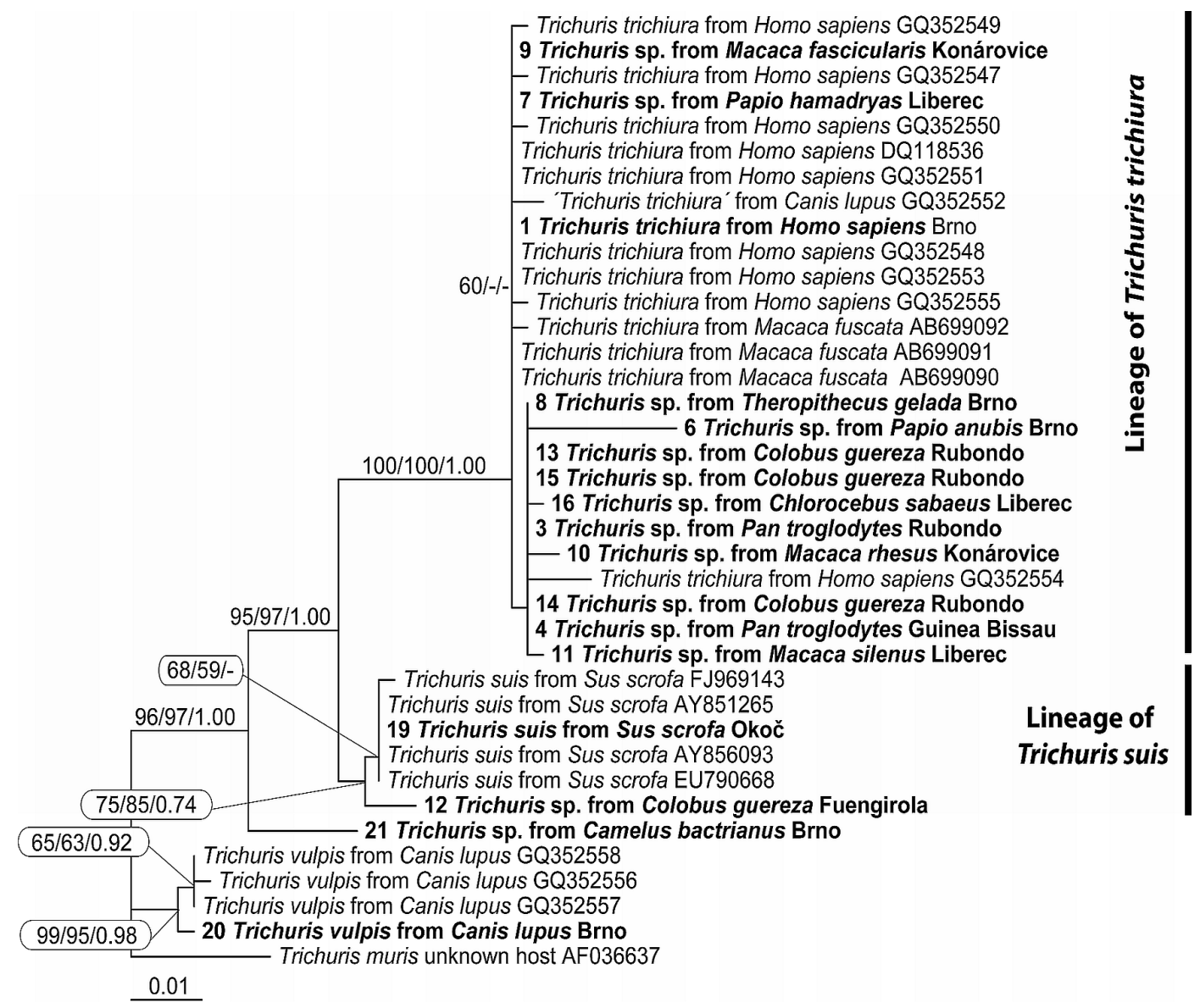

Fig. 3. Maximum parsimony phylogenetic tree as inferred from partial $18 \mathrm{~S}$ rRNA gene sequences. Numbers above branches indicate MP bootstrap support (1000 replicates)/ML bootstrap support (1000 replicates)/Bayesian posterior probabilities (see Methods for details). Sequences newly reported in this study are bold typed and basic clades are highlighted. Tree was rooted with Trichuris muris (Schrank, 1788) (AF036367).

tions were discarded as a 'burnin'. The unrooted combined tree is shown (Fig. S3).

\section{RESULTS}

The described method of DNA isolation from whipworm eggs proved to be rather successful as we were able to retrieve DNA from almost $90 \%$ of the samples of eggs collected (negative data not shown). The lengths of sequences were 550-620 bp for ITS2, 669-750 bp for 18S rDNA, and 390-430 bp for coxl. Due to a low amount of DNA from the eggs in some isolates, we obtained 13 ITS2, 17 18S rDNA and 9 cox 1 amplicons. The 18S and ITS2 alignments were over 518 and 327 positions, respectively, whereas the coxl alignment was comprised of 124 amino acids. These datasets were analysed separately and also in a concatenated alignment. This alignment contained 525 18S rDNA positions, 354 ITS2 positions and 372 cox 1 positions (1251 positions in total).

The primary Kalign alignment of ITS2 was 1425 nucleotides long. Manual editing (deleting gaps and ambiguously aligned regions) produced alignment of 327 nucleotides, alternative Gblock selection resulted in alignment 383 nucleotides long. When the webPRANK approach was applied to the dataset, it produced primary alignment consisting of 1465 nucleotides; when the Gblock was used to delete gaps (primary alignment contained positions unusable for phylogenetic analysis, mainly due to the occurrence of gaps), the resulting alignment contained 276 nucleotides. However, in spite of the mentioned differences, all alignments showed almost identical but weakly supported topologies (Figs. 2, S1). The primary alignment of $18 \mathrm{~S}$ rDNA consisted of 555 nucleotides, which was reduced by manual editing to 518 nucleotides long alignment. The primary alignment constructed by webPRANK was 545 nucleotides long and for tree computation, it was reduced using Gblock to 523 nucleotides (primary alignment contained positions unusable for phylogenetic analysis, mainly due to the occurrence of gaps in a fraction over $80 \%$ ). Trees inferred from the mentioned alignments do not display substantial differences in topology.

ML analysis based on ITS2 revealed three basic lineages within the genus Trichuris (Fig. 2). The first lineage was mostly composed of whipworms from pigs (classified as $T$. suis; lineage of $T$. suis), with a separate subclade containing isolates from man and various primates (a novel subclade from primates; see Fig. 2).

The second lineage contained sequences of Trichuris classified as T. trichiura (Fig. 2) isolated from humans and non-human Old World primates. This clade contained a large group of almost identical whipworm se- 


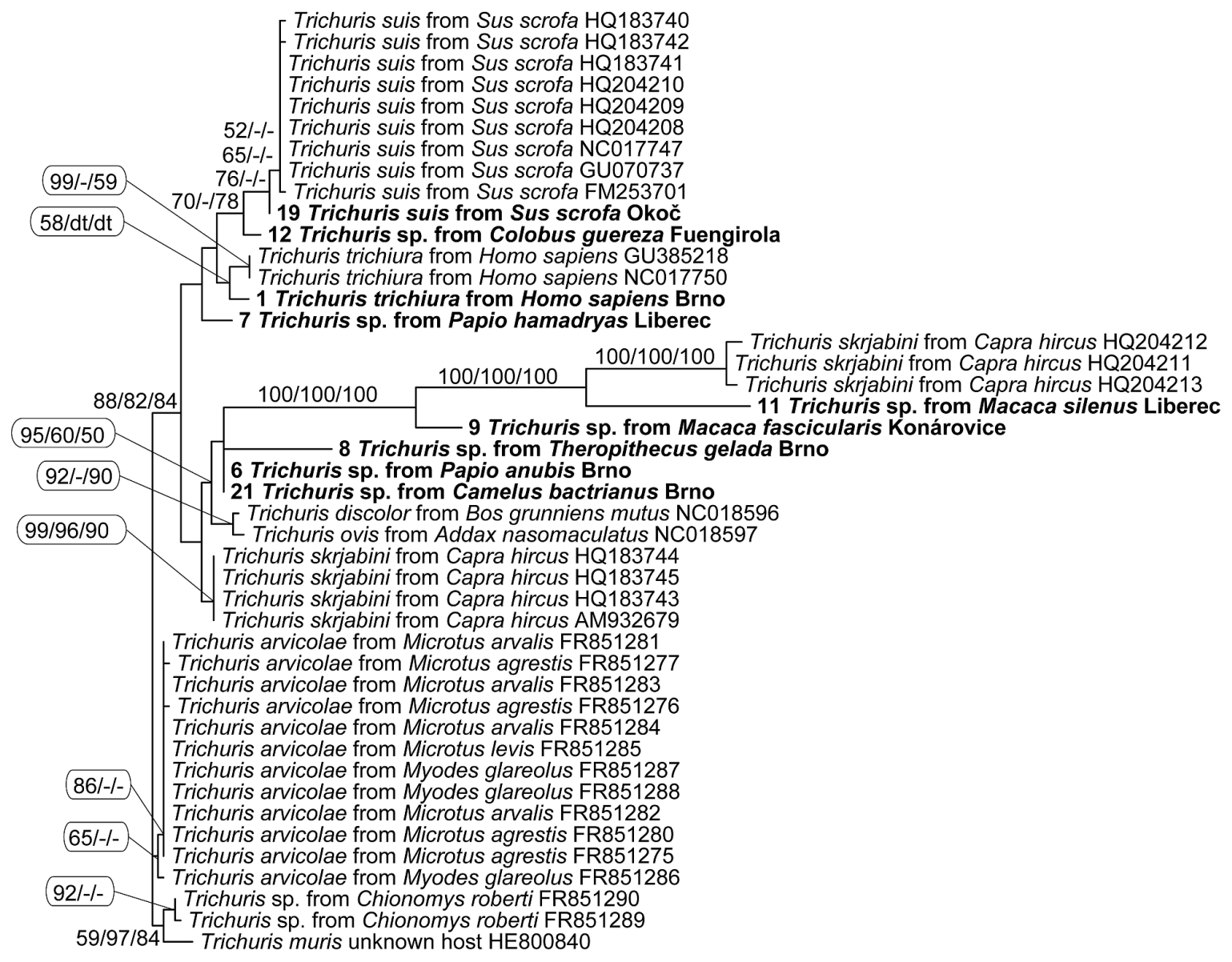

0.1

Fig. 4. Unrooted maximum parsimony coxl tree of the genus Trichuris Roederer, 1761 as inferred from partial coxl amino acid sequences. Numbers above branches indicate MP bootstrap support (1 000 replicates)/ML bootstrap support (1 000 replicates)/NJ bootstrap support (1000 replicates). Sequences newly reported in this study are bold typed.

quences from humans and primates and also six relatively distant sequences from Macaca fascicularis (isolate 9), M. fuscata Blyth (AB586133), Papio anubis (isolate 6), Theropithecus gelada (isolate 8), Chlorocebus aethiops pygerythrus (isolate 17) and Nomascus gabriellae (isolate 5).

The third lineage comprised whipworms from rodents and carnivores containing several morphologically and biologically distinguishable species [T. muris, T. arvicolae and T. vulpis (Frölich, 1789)].

The tree based on partial 18S rDNA sequences (Fig. 3) contained a substantially lower number of sequences. However, isolates from humans and non-human primates again formed two distinct clades. The first clade was composed of genetically almost identical isolates classified as Trichuris trichiura, including sequences from Macaca silenus (isolate 11), Chlorocebus sabaeus (isolate 16) and one isolate from dog (a sequence of ambiguous origin), whereas the isolate from Colobus guereza kikuyuensis (isolate 12) is placed at the root of the second T. suis clade. Both clades of T. trichiura and $T$. suis formed sister groups, similar to analyses based on ITS2 data. The Trichuris specimen from camel formed a basal clade to both of the mentioned groups. The last clade comprised T. vulpis and T. muris isolates (Fig. 3). No substantial differences were found between trees inferred from manually edited alignments (shown in Figs. 2, 3) and those obtained using probabilistic webPRANK and edited by Gblocs to delete gaps from the alignment (see Figs. S1, S2). Composition of main clades is identical, only some particular positions of closely related isolates changed within the same clade.

The only discrepancies between trees inferred from ITS2 and 18S regions are in divergent positions of isolates 11 (Macaca silenus Liberec) and 16 (Chlorocebus sabaeus Liberec). These specimens are both classified as T. suis in the ITS2 tree (specifically in a novel subclade from primates; see Fig. 2), while $18 \mathrm{~S}$ placed it within T. trichiura clade (Fig. 3).

Tree topology based on the coxl appears to be very unstable, probably because of the low information content of the partial sequence. Analysis of the coxl from species of Trichuris (Fig. 4) divided isolates from humans and non-human primates into two clades representing T. trichiura and T. suis, with the isolate from Papio hamadryas (isolate 7) on the basis of clades. However, the positions of more individual isolates, as revealed by coxl (Fig. 4), differed from those observed in ITS2 (Fig. 2), 18S rDNA (Fig. 3) and extended cox 1 analyses (Fig. 5) in three respects. 


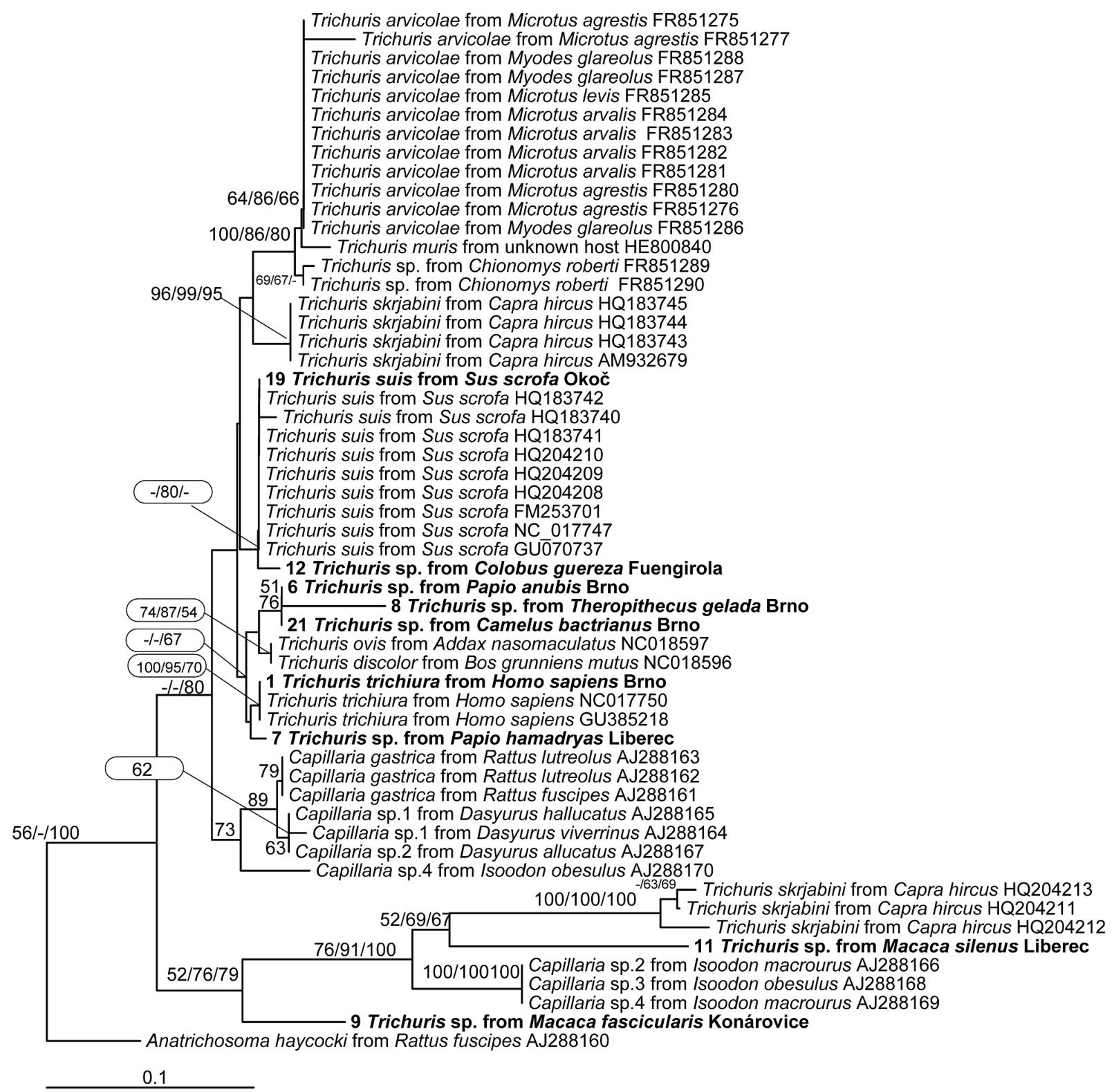

Fig. 5. Neighbor joining tree (by AsaturA, which is designed to deal with amino acid saturation) as inferred from coxl amino acid sequences using an extended data set. Numbers above branches indicate NJ bootstrap support (1 000 replicates)/ML bootstrap support (1000 replicates)/MP bootstrap support (1000 replicates). Sequences newly reported in this study are bold typed. Tree is rooted to Anatrichosoma haycocki Spratt, 1982 (AJ288160).

(1) The isolate from Colobus guereza kikuyuensis (isolate 12) appeared in two different positions according to the used method of tree reconstruction: (i) NJ analysis of the amino acid alignment (AsaturA) showed the unsupported affiliation of this isolate with T. suis; (ii) ML (PhyML) placed it in an unsupported position at the root of the $T$. trichiura cluster. Analysis based on the 18S rDNA (Fig. 3) and the combined dataset of all three genes confirmed the relationship of this isolate to T. suis (see Fig. S3); the ITS2 tree shows a very well supported affiliation of the Colobus guereza isolate to a diverse clade closely related to $T$. suis (Fig. 2).

(2) Trichuris from Macaca fascicularis (isolate 9) is in the ITS2 tree branching together with isolate from Papio anubis (isolate 6) within the T. trichiura clade. While $18 \mathrm{~S}$ rDNA-based analysis placed these isolates as relative to
T. trichiura as well, the cox 1 tree grouped isolate 6 (from P. anubis) with the Theropithecus gelada (isolate 8) and Macaca silenus (isolate 11) isolates in a substantially different position in close proximity to T. skrjabini (Fig. 4) and in extended coxl analyses to the genus Capillaria (Fig. 5). Thus, species of Capillaria appeared inside the genus Trichuris, making the genus Trichuris polyphyletic (Fig. 5).

(3) In the extended coxl tree (Fig. 5) within the T. trichiura clade, two subclades were formed: the first included isolates from humans and Papio hamadrays (isolate 7), whereas the second one was formed by isolates from P. anubis (isolates 6), T. gelada (isolate 8) and ruminants (Trichuris ovis, T. discolor, Trichuris sp. from camel). The ambiguity of cox 1 phylogeny of the genus Trichuris is underscored by the unexpected positions of T. skrjabi- 
$n i$, which clustered in two substantially different clades (Fig. 5).

The concatenated tree constructed from partial sequences of the 18S, ITS2 and cox 1 sequences supported the division of the primate isolates into two clades (see Fig. S3). The position of whipworm isolates from Macaca silenus (11) and C. guereza kikuyuensis (12) within the T. suis clade and other primate isolates to the $T$. trichiura clade are congruent with the ITS2 analysis.

\section{DISCUSSION}

Similar to other nematodes, whipworm species are described and distinguished on the basis of morphological and biometrical features, the host(s) they infect and/ or their geographical origin (Robles 2011, Torres et al. 2011). However, adherence to the morphological species concept requires the examination of well-preserved mature nematodes of both sexes, which are almost inaccessible from endangered mammals such as primates. Misleading classification could also obscure the zoonotic potential of whipworms.

Our study addresses to some extent the molecular diversity of isolates of Trichuris using partial sequences of nuclear and mitochondrial genomic sequences. The majority of phylogenetic studies on whipworms have been based on analyses of ITS1-5.8S-ITS2 rDNA, which revealed the relationships of rodent- and ruminant-infecting species of Trichuris (e.g. Cutillas et al. 2004, Callejón et al. 2012). However, the occurrence of more variants of RNA genes, including ITS2 region (Nissen et al. 2012), makes their use in solving the phylogeny of Trichuris much less informative, especially in organisms with unknown ploidy.

Nissen et al. (2012) detected two variants of ITS2 sequences in some individuals of Trichuris isolated from humans, one showing an affiliation with $T$. suis whereas the other clustering with $T$. trichiura. They proposed existence of two distinct genotypes in humans, which were probably due to cross-infections of man with $T$. suis and consequent hybridisation of these two species.

In contrast, Ravasi et al. (2012) suggested that these results were due to cross-contamination with genomic DNA of T. suis. Being aware of these issues, we have tested randomly selected isolates of Trichuris for the presence of gene variants by cloning and sequencing individual clones. Furthermore, the quality of the sequence reads from direct sequencing of PCR products was assessed (data not shown). We found no indication for more variants of the target genes or contamination.

Our phylogenetic analyses of whipworms from primates have shown the presence of two independent evolutionary lineages: (i) the T. trichiura clade and (ii) a clade primarily containing $T$. suis. This trend was also observed in isolates from humans, non-human primates and pigs (Liu et al. 2012, Nissen et al. 2012, Ravasi et al. 2012, Callejón et al. 2013). The majority of sequences (both from captive and free-ranging primates) within the T. trichiura clade can be classified as T. trichiura sensu stricto. Such an arrangement supports the zoonotic potential of $T$. trichiura and its ability to infect a broader range of primates (Munene et al. 1998, Phillips et al. 2004, Lee et al. 2010).

The $T$. suis clade, including whipworm sequences from pigs and primates, further branched into two subclades. The isolates from European, Asian and African domestic pigs and humans formed a highly geneticaly uniform group that could be unequivocally considered as $T$. suis sensu stricto. The isolates from one human and Old World primates of the second subclade might represent different taxon/taxa of Trichuris. Obviously, the isolates referred to as T. trichiura isolated from humans (Ravasi et al. 2012) and Nomascus gabriellae (see Cutillas et al. 2009) should be reclassified as was performed by isolate from Colobus guereza kikuyuensis (see Cutillas et al. 2014).

To overcome troubles with nuclear rDNA-based phylogenetic analyses, mitochondrial DNA sequences have become widely used (e.g. Blouin et al. 1998, Peng et al. 2007, Jex et al. 2009), allowing high resolution in deep analyses of closely related lineages (Callejón et al. 2012). However, when comparing rDNA and coxl data alone, support of branches is higher in trees based on nuclear data. Moreover, we obtained different topologies of some isolates: ITS2 placed an isolate from Macaca silenus (isolate 11) and Chlorocebus sabaeus (isolate 16) to the sister position to $T$. suis, whereas $18 \mathrm{~S}$ rDNA analysis shows an affiliation of these isolates to T. trichiura. Phylogenetic position (NJ and ML tree) of the isolate from M. silenus (isolate 11) as inferred from coxl (Fig. 4) was among the isolates from ruminants and appeared in wider phylogenetic context in close proximity to the genus Capillaria (Fig. 5).

The presence of the genera Capillaria and Anatrichosoma in cox 1 analyses revealed an unexpected finding: isolates of Capillaria branched inside those of Trichuris, making thus this genus polyphyletic. Especially, Trichuris isolates from M. silenus (11) and Macaca fascicularis (9) formed a highly diverse clade together with three isolates of T. skrjabini from Capra hircus (HQ204211; HQ204212; HQ204213) and three sequences from Capillaria spp. The rest of sequences of Capillaria spp. branched at the root of the core taxa of Trichuris, which are defined here as species of Trichuris after exclusion of the diverse basal clade (see Fig. 5).

Similarly, T. skrjabini appears in two different positions in the cox 1 tree, three isolates were placed in the diverse basal cluster, while four others are in an advanced position as a sister group to T. arvicolae. Such a topology can be caused by the maternal heredity of mitochondrial genes (see Fig. S4 for details) or these isolates from a presumably diverse clade (HQ204211; HQ204212; HQ204213) were simply misclassified. However, since sequences HQ183745 and HQ204213 were obtained from a single isolate (GenBank information), maternal mitochondrial heredity and hybridisations might have affected the phylogeny of the genus. Although mitochondrial DNA is proposed to be used for phylogenetic and interspecific analyses in some nematodes (Liu et al. 2012, Jabbar et al. 2013), discrepancies in our analyses may argue against its use. 
Although these discrepancies can also result from the low number of available species in both genera Trichuris and Capillaria, from the erroneous determination of species of Capillaria or from the presence of vestigial mitochondrial genes recently transferred to the nucleus (the so-called 'numts') (Richly and Leister 2004), we prefer a scenario involving hybridisation, ancient gene or genome duplication (Cardoso et al. 2006) or ancestral polymorphism (as explained in Fig. S5). It should be also noted that due to low sequence variability within mitochondrial coxl DNA, the use of a partial gene region (Liu et al. 2013) is likely not efficient to solve phylogenetic relationships among Trichuris spp. and related species. This notion is supported by the high variability of the topologies obtained by the three methods (see Figs. 4, 5). However, all used methods revealed the polyphyletic nature of Trichuris.

We combined nuclear and organellar DNA markers, which is recommended as a suitable phylogenetic approach, as the differences between the markers can enrich the interpretation of the evolutionary history of nematodes (e.g. Rubinoff and Holland 2005, Zarlenga et al. 2006). Our study represents the first attempt to join 18S, ITS2 and coxl markers in a concatenated analysis of Trichuris. However, the resultant dataset was drastically reduced due to the paucity of sequences in the GenBank Database. The isolates were divided into two groups roughly comprising human-primate and pig isolates, with two isolates from primates (C. guereza kikuyuensis and M. silenus) branching at the root of the pig clade (see Fig. S3).

So far, the whipworms referred as T. trichiura or Trichuris sp. were reported from 10 families of primates across three continents (e.g. Michaud et al. 2003, Phillips et al. 2004, Cutillas et al. 2009, Clough 2010, Raharivololona and Ganzhorn 2010). Considering the diversity of Trichuris spp. affecting other mammalian groups, such as rodents and ruminants (e.g. Cutillas et al. 2004, Robles 2011), it is unlikely that primates are infected with only one or two species of whipworms, as suggested in previous studies (Ravasi et al. 2012, Liu et al. 2013, Cutillas et al. 2014).

Our ITS2 analysis revealed three genetically different isolates of Trichuris from baboons scattered in both of the described major lineages. Trichuris cynocephalus was, until this year (Cutillas et al. 2014), the only species other than $T$. trichiura named from the primate suborder Haplorhini. This taxon was described based on subtle differences in whipworm morphology from captive yellow baboons Papio cynocephalus Linnaeus, by Khera (1951).

However, despite our morphological analyses of an isolate from Papio hamandryas (data not shown), we are not able to associate any particular sequences from baboons with $T$. cynocephalus. Thus, the question of the identity and validity of this species needs the study of more isolates from free-ranging Papio spp. Obviously, morphological analyses of adult whipworms are an essential complement to the molecular data as shown by Cutillas et al. (2014).
Evidence for more species of Trichuris in primates also has practical implications for epidemiology. A great deal of whipworms eggs found in the faeces of non-human primates have been classified as T. trichiura (e.g. Ooi et al. 1993, Reichard et al. 2008, Lee et al. 2010). Such a tentative placement may imply the broad zoonotic cross-transmission between non-human primates and humans (e.g. Munene et al. 1998, Chapman et al. 2006), which can be misleading in most cases. In contrast, limited experimental data have demonstrated the zoonotic transmission of T. trichiura between non-human primates and man (Horii and Usui 1985, Imada et al. 1986).

This finding is consistent with the close proximity of some of our isolates of Trichuris from primates and human-originated T. trichura (Fig. 2). However, most of the Trichuris isolates sequenced are from captive hosts and various Trichuris spp. might differ in their host specificity/preferences in free ranging populations (see Poulin and Keeney 2008). In the wild, various species of primates often overlap in their ranges and share habitats and/or foraging sites. Experimental transmission studies in primates to address host specificity of parasites are almost impossible, but careful selection of model field sites and a survey of the genetic diversity of species of Trichuris in such natural multi-primate communities can answer ongoing questions about host specificity.

Although named almost 250 year ago, whipworms of man and primates deserve more attention from epidemiologists. The obvious existence of more different species in man, broad distribution of trichuriasis in humans together with unresolved zoonotic aspects of 'new' species call for further research to address the diversity of whipworms in these hosts.

Acknowledgements. We are grateful to the zoo keepers, curators and veterinarians who have provided us with samples of captive primates, namely from zoos in Liberec, Brno, Bratislava, Antwerp and Fuengirola. Furthermore, we acknowledge the staff of BioTest s.r.o., Konárovice, and a pig farm in Okoč, both in the Czech Republic. Samples from wild primates were obtained from Rubondo Island and approved by the Tanzania Wildlife Institute, Tanzania Commission for Science and Technology and Tanzania National Parks. We express special thanks to the Rubondo Island National Park management and also to the Guinea-Bissau environmental agency (IBAP) for permission to work in CNP and logistical support. This publication is an outcome of the HPL-lab, Laboratory for Infectious Diseases Common to Humans and (non-Human) Primates. This study was supported by the project "CEITEC- Central European Institute of Technology" (CZ.1.05/1.100/02.0068) from European Regional Development Fund, the European Social Fund and state budget of the Czech Republic (project no. CZ.1.07/2.3.00/20.0300), by grants from the Grant Agency of the Czech Republic (524/06/0264 and 206/09/0927), by the grant of IGA UVPS Brno (IGA 77/2007/ FVL), by the institutional support from Institute of Parasitology (RVO: 60077344) and a grant from the Ministry of Science and Technology (CGL2008-01459/BOS) of Spain. 


\section{REFERENCES}

Anderson R.C. 2000: Nematode Parasites of Vertebrates. Their Development and Transmission. Second Edition. CABI Publishing, Wallingford, $672 \mathrm{pp}$.

Ashford R.W., Reid G.D.F., Wrangham R.W. 2000: Intestinal parasites of the chimpanzee Pan troglodytes in Kibale Forest, Uganda. Ann. Trop. Med. Parasitol. 94: 173-179.

Blouin M.S., Yowell C.A., Courtney C.H., Dame J.B. 1998: Substitution bias, rapid saturation, and the use of mtDNA for nematode systematics. Mol. Biol. Evol. 15: 1719-1727.

Callejón R., De Rojas M., Ariza C., Ubeda J.M., Guevara D.C., Cutillas C. 2009: Cytochrome oxidase subunit 1 and mitochondrial 16S rDNA sequences of Trichuris skrjabini (Trichocephalida: Trichuridae). Parasitol. Res. 104: 715-716.

Callejón R., De Rojas M., Feliú C., Balao F., Marrugal A., Henttonen H., Guevara D., Cutillas C. 2012: Phylogeography of Trichuris populations isolated from different Cricetidae rodents. Parasitology 139: 1795-1812.

Callejón R., Nadler S., De Rojas M., Zurita A., Petrášová J., Cutillas C. 2013: Molecular characterization and phylogeny of whipworm nematodes inferred from DNA sequences of cox1 mtDNA and 18S rDNA. Parasitol. Res. 112: 3933-3949.

Cardoso J.C., Pinto V.C., Vieira F.A., Clark M.S., Power D.M. 2006: Evolution of secretin family GPCR members in the metazoa. BMC Evol. Biol. 6: 108.

Castresana J. 2000: Selection of conserved blocks from multiple alignments for their use in phylogenetic analysis. Mol. Biol. Evol. 17: 540-552.

Chabaud A.G., Brygoo E.R., Petter A.J. 1964: Les nématodes parasites de lémuriens malgaches. V. Nématodes de Daubentonia madagascariensis. Vie Milieu 17, Suppl.: 205-212.

Chapman C.A., Gillespie T.R., Speirs M., Holland T., AusTAD K. 2006: Life on the edge: a comparison of primate gastrointestinal parasites from forest edge and interior groups. Am. J. Primatol. 68: 397-409.

Clough D. 2010: Gastro-intestinal parasites of red-fronted lemurs in Kirindy Forest, Western Madagascar. J. Parasitol. 96: 245251.

Cutillas C., Callejón R., De Rojas M., Tewes B., Ubeda J.M., Ariza C., Guevara D.C. 2009: Trichuris suis and Trichuris trichiura are different nematode species. Acta Trop. 111: 299-307.

Cutillas C., De Rojas M., Zurita A., Oliveros R., Callejón R. 2014: Trichuris colobae n. sp. (Nematoda: Trichuridae), a new species of Trichuris from Colobus guereza kikuyensis. Parasitol. Res. 113: 2725-2732.

Cutillas C., Oliveros R., De Rojas M., Guevara D.C. 2004: Determination of Trichuris skrjabini by sequencing of the ITS15.8S-ITS2 segment of the ribosomal DNA: comparative molecular study of different species of trichurids. J. Parasitol. 90; $648-652$.

Gouy M., Guindon S., Gascuel O. 2010: SeaView version 4: a multiplatform graphical user interface for sequence alignment and phylogenetic tree building. Mol. Biol. Evol. 27: 221-224.

Guindon S., Gascuel O. 2003: A simple, fast, and accurate algorithm to estimate large phylogenies by maximum likelihood. Syst. Biol. 52: 696-670.

Hall T.A. 1999: BioEdit: a user-friendly biological sequence alignment editor and analysis program for Windows 95/98/NT. Nucl. Acids Symp. Ser. 41: 95-98.

HoRII Y., Usui M. 1985: Experimental transmission of Trichuris ova from monkeys to man. Trans. R. Soc. Trop. Med. Hyg. 79: 423.

IMADA I., HoRII Y., Usui M. 1986: [The experiment of artificial infection to men and monkeys by eggs of Trichuris trichiura from Japanese monkey.] Jpn. J. Parasitol. 29: S30 (In Japanese.).

Jabbar A., Jex A.R., Mohandas N., Hall R.S., LittleWOOD D.T., Gasser R.B. 2013: The mitochondrial genome of
Aelurostrongylus abstrusus - diagnostic, epidemiological and systematic implications. Gene 516: 294-300.

Jex A.R., Waeschenbach A., Hu M., van Wyk J.A., Beveridge I., Littlewood D.T.J., Gasser R.B. 2009: The mitochondrial genomes of Ancylostoma caninum and Bunostomum phlebotomum - two hookworms of animal health and zoonotic importance. BMC Genomics 10: 79.

Kalema-Zikusoka G., Rothman J.M., Fox M.T. 2005: Intestinal parasites and bacteria of mountain gorillas (Gorilla beringei beringei) in Bwindi Impenetrable National Park, Uganda. Primates 46: 59-63.

Khera S. 1951: Trichuris cynocephalus n. sp. (family Trichinellidae Stiles \& Crane, 1910: Nematoda) from the Abyssinian baboon, Cynocephalus hamadryas Linn. Ind. J. Helminthol. 3: 87-92.

Knight R.A. 1983: Trichuris odocoileus sp. n. (Nematoda: Trichuridae) from white-tailed deer, Odocoileus virginianus, in southeastern U.S., and a key to trichurids in North American ruminants. J. Parasitol. 69: 1156-1159.

Labes E.M., Hegglin D., Grimm F., Nurcahyo W., Harrison M.E., Bastian M.L., Deplazes P. 2010: Intestinal parasites of endangered orangutans (Pongo pygmaeus) in Central and East Kalimantan, Borneo, Indonesia. Parasitology 137: 123-135.

Lassmann T., Sonnhammer E.L. 2005: Kalign - an accurate and fast multiple sequence alignment algorithm. BMC Bioinformatics 6: 298 .

Lee J., Kang S., Kim N., Lee C., Ahn K., Kwon H., Park C., KIM S. 2010: Investigation of helminths and protozoans infecting old world monkeys: captive vervet, cynomolgus, and rhesus monkeys. Kor. J. Vet. Res. 50: 273-277.

Lim Y.A., Ngui L.R., Shukuri J., Rohela M., Mat Naim H.R. 2008: Intestinal parasites in various animals at a zoo in Malaysia. Vet. Parasitol. 157: 154-159.

Liu G.H., Gasser R.B., Nejsum P., Wang Y., Chen Q., Song HQ, ZHU X.Q. 2013: Mitochondrial and nuclear ribosomal DNA evidence supports the existence of a new Trichuris species in the endangered françois' leaf-monkey. PLoS ONE 8: e66249.

Liu G.H., Gasser R.B., Su A., Nejsum P., Peng L., Lin R.Q., Li M.W., Xu M.J., ZHU X.Q. 2012: Clear genetic distinctiveness between human- and pig-derived Trichuris based on analyses of mitochondrial datasets. PLoS Negl. Trop. Dis. 6: e1539.

Logan C., Hanks M.C., Noble-Topham S., Nallainathan D., Provart N.J., Joyner A.L. 1992: Cloning and sequencing comparison of the mouse, human, and chicken engrailed genes reveal potential function domains and regulatory regions. Dev. Genet. 13: 345-358.

Melfi V., Poyser F. 2007: Trichuris burdens in zoo-housed Colobus guereza. Int. J. Primatol. 28: 1449-1456.

Michaud C., Tantalean M., Ique C., Montoya E., Gozalo A. 2003: A survey for helminth parasites in feral New World non-human primate populations and its comparison with parasitological data from man in the region. J. Med. Primatol. 32: 341-345.

Mul I.F., Paembonan W., Singleton I., Wich S.A., van BolHUIS H.G. 2007: Intestinal parasites of free-ranging, semicaptive and captive Pongo abelii in Sumatra, Indonesia. Int. J. Primatol. 28: 407-420.

Munene E., Otsyula M., Mbaabu D.A., Mutahi W.T., MuRIUKi S.M.K., Muchemi G.M. 1998: Helminth and protozaon gastrointestinal tract parasites in captive and wild-trapped African non-human primates. Vet. Parasitol. 78: 195-201.

Nissen S., Al-Jubury A., Hansen T.V., Olsen A., Christensen H., Thamsborg S.M., Nejsum P. 2012: Genetic analysis of Trichuris suis and Trichuris trichiura recovered from humans and pigs in a sympatric setting in Uganda. Vet. Parasitol. 188: 68-77.

Ooi H.K., Tenora F., Itoh K., Kamiya M. 1993: Comparative study of Trichuris trichiura from non-human primates and from 
man, and their difference with T. suis. J. Vet. Med. Sci. 55: 363 366.

Peng W., Yuan K., Hu M., Gasser R.B. 2007: Recent insights into the epidemiology and genetics of Ascaris in China using molecular tools. Parasitology 134: 325-330.

Petrášová J., Modrý D., Huffman M.A., Mapua M.I., Bobáková L., Mazoch V., Singh J., Kaur T., Petrželková K.J. 2010: Gastrointestinal parasites of indigenous and introduced primate species of Rubondo Island National Park, Tanzania. Int J. Primatol. 31: 920-936.

Phillips K.A., Haas M.E., Grafton B.W., Yrivarren M. 2004: Survey of the gastrointestinal parasites of the primate community at Tambopata National Reserve, Peru. J. Zool. 264: 149-151.

Poulin R., Keeney D. 2008: Host specificity under molecular and experimental scrutiny. Trends Parasitol. 24: 24-28.

Raharivololona B.M., Ganzhorn J.U. 2010: Seasonal variations in gastrointestinal parasites excreted by the gray mouse lemur Microcebus murinus in Madagascar. Endanger. Species Res. 11: 113-122.

Ravasi D.F., O’Riain M.J., Davids F., Illing N. 2012: Phylogenetic evidence that two distinct Trichuris genotypes infect both humans and non-human primates. PLoS ONE 7: e44187.

Reichard M.V., Wolf R.F., Clingenpeel L.C., Doan S.K., Jones A.N., Gray K.M. 2008: Efficacy of fenbendazole formulated in a commercial primate diet for treating specific pathogen-free baboons (Papio cynocephalus anubis) infected with Trichuris trichiura. J. Am. Assoc. Lab. Anim. Sci. 47: 51-55.

Richly E., LeISTER D. 2004: NUMTs in sequenced eukaryotic genomes. Mol. Biol. Evol. 21: 1081-1084.

Robles M.R. 2011: New species of Trichuris (Nematoda: Trichuridae) from Akodon montensis Thomas, 1913, of the Paranaense Forest in Argentina. J. Parasitol. 97: 319-327.

Ronquist F., Teslenko M., van der Mark P., Ayres D.L., Darling A., Hohna S., Larget B., Liu L., Suchard M.A., HuElSENBECK J.P. 2012: MrBayes 3.2: efficient Bayesian phylogenetic inference and model choice across a large model space. Sys. Biol. 61: 539-542.

Rubinoff D., Holland B.S. 2005: Between two extremes: mitochondrial DNA is neither the panacea nor the nemesis of phylogenetic and taxonomic inference. Syst. Biol. 54: 952-961.

Sá R.M., Petrášová J., Pomajbíková K., Profousová I., Petrželková K.J., Sousa C., Cable J., Bruford M.W.,
MoDRÝ D. 2013: Gastrointestinal symbionts of chimpanzees in Cantanhez National Park, Guinea-Bissau with respect to habitat fragmentation. Am. J. Primatol. 75: 1032-1041.

Sambrook J., Russell D.W. 2006: Purification of nucleic acids by extraction with phenol : chloroform. Cold Spring Harb Protoc. http://cshprotocols.cshlp.org/content/2006/1/pdb.prot4455

SheATher A.L. 1923: The detection of intestinal protozoa and mange parasites by a flotation technique. J. Comp. Pathol. 36: 266-275.

ŠKeří́KovÁ A., HYPŠA V., Scholz T. 2004: A paraphyly of the genus Bothriocephalus Rudolphi, 1808 (Cestoda: Pseudophyllidea) inferred from internal transcribed spacer-2 and 18S ribosomal DNA sequences. J. Parasitol. 90: 612-617.

ŠPAKUlová M. 1994: Discriminant analysis as a method for the numerical evaluation of taxonomic characters in male trichurid nematodes. Syst. Parasitol. 29: 113-119.

Stamatakis A. 2014: RAxML version 8: a tool for phylogenetic analysis and post-analysis of large phylogenies. Bioinformatics 1: $1312-1313$.

Swofford D.L. 2002: PAUP* Phylogenetic Analysis Using Parsimony, Version 4. Sinauer Associates, Sunderland, MA.

Talavera G., Castresana J. 2007: Improvement of phylogenies after removing divergent and ambiguously aligned blocks from protein sequence alignments. Syst. Biol. 56: 564-577.

Torres E.J.L., Nascimento A.P.F., Menezes A.O., Garcia J., dos Santos M.A.J., Maldonado Jr. A., Miranda K., LanFREDI R.M., DE SOUZA W. 2011: A new species of Trichuris from Thrichomys apereoides (Rodentia: Echimyidae) in Brazil: morphological and histological studies. Vet. Parasitol. 176: 226-235.

Van de Peer Y., Frickey T., Taylor J., Meyer A. 2002: Dealing with saturation at the amino acid level: a case study based on anciently duplicated zebrafish genes. Gene 295: 205-211.

White T.J., Bruns T., Lee S., TAYlor J. 1990: PCR Protocols: A Guide to Methods and Application. Academic Press, San Diego, California, $482 \mathrm{pp}$.

Zarlenga D.S., Rosenthal B.M., La Rosa G., Pozio E., HoberG E.P. 2006: Post-miocene expansion, colonization, and host switching drove speciation among extant nematodes of the archaic genus Trichinella. Proc. Natl. Acad. Sci. USA 103: 7354-7359.

Cite this article as: Doležalová J., Oborník M., Hajdušková E., Jirků M., Petrželková K.J., Bolechová P., Cutillas C., Callejón R., Jaroš J., Beránková Z., Modrý D.: How many species of whipworms do we share? Whipworms from man and other primates form two phylogenetic lineages. Folia Parasitol. 62: 063. 